\title{
A Joint Timing and Fractionally-Space Blind Equalization Algorithm
}

\section{Zheng Dong ${ }^{\text {a }}$, Kexian Gong ${ }^{\text {b }}$, Lindong Ge}

Institute of Zhengzhou Information science and technology, Zhengzhou 450002, China

\author{
a DZ1984114@163.com, b ggkx@163.com
}

Key words: blind equalization, fractionally-spaced, CMA, timing

\begin{abstract}
In this paper, we proposed a joint timing and fractionally-spaced blind equalization algorithm. It adopts Gardner algorithm instead of simple down-sampling in conventional fractionally-spaced equalization in order to overcome the problem of that the filter can't compensate timing error. The simulations show that the performance of the proposed fractionallyspaced equalization algorithm is better than CMA in different SNR and in different number of channel taps.
\end{abstract}

\section{Introduction}

Fractionally-spaced equalization (FSE) is compared with the traditional symbol-spaced equalization (SRE), it can sample the received signal as the greater rate of symbol rate. Insensitive to sampling phase, functional timing and compensation for serious sideband distortion are the outstanding advantages of the FSE. Further study suggests that a limited length FSE can completely equalize a FIR channel, while SRE requires an infinite length filter to equalize a FIR channel perfectly. FSE refers to the fractionally-spaced blind equalization structure; FSE-CMA is one of the most popular structures of fractionally-spaced blind equalization. FSE-CMA adopts the algorithms of CMA coefficients updating, the consequence of simulations shows that it can play a perfect balanced effect and it can better convergence especially for the MPSK signal.

\section{Multi-Channel System Model of Fractionally-Spaced Equalization (FSE)}

Many authors have studied in depth on the structure of FSE; the multichannel and single channel system model of FSE has been studied in [1][2][3]. Channel system model of FSE can use single symbol rate to express the full signal transmission process, every sample points of each symbol can be viewed as a sub-channel, and therefore FSE can be transformed as multi-channel system model $^{[4][5]}$.

Supposing that the sending symbol sequence $\left\{s_{n}\right\}$ is transferred through a linear time-invariant (LTI) channel, the output of the fractionally-spaced channel could be expressed as,

$$
r_{n}=r\left(n \frac{T}{P}\right)=\sum_{i} s_{i} h\left(n \frac{T}{P}-i T\right)+v\left(n \frac{T}{P}\right)=\sum_{i} s_{i} h_{n-i P}+v_{n}
$$

Where $h_{n}$ refers to fractionally-spaced sampling points of the channel impulse response, $v_{n}$ refers to fractionally-spaced sampling points of noise. For the finite impulse response channel, the coefficient of fractionally-spaced channel can be expressed as vector format $\boldsymbol{h}=\left(h_{0}, h_{1}, h_{2}, \cdots, h_{\left(L_{h}+1\right) P-1}\right)^{T}$. Where $L_{h}$ refers to the length of the channel impulse response which sampling space is the rate of symbol period.

Fractional sampling rate model can be equivalent to a single input multiple output (SIMO) channel model (as shown in Fig. 1). The impulse response one of the sub-channel $p$ $(p=\{1,2, \cdots, P\})$, recorded as $h_{n}^{(p)}=h_{(n+1) P-p}$, could be obtained through the over-sampling vector $\boldsymbol{h}$. The output of the corresponding sub-channel could be expressed as, 


$$
r_{n}^{(p)}=\sum_{i=0}^{L_{h}} s_{i} h_{n-i}^{(p)}+v_{n}^{(p)}
$$

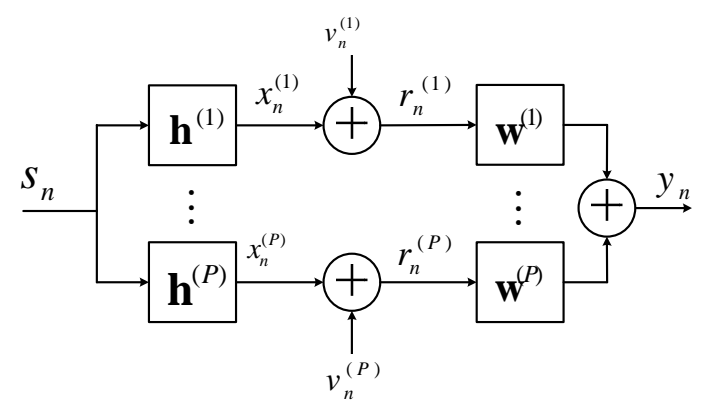

Fig. 1 multi-channel system model of the $T / P$ interval equalizer

As shown in the figure of the equalization model above, the corresponding coefficient $h_{n}^{(p)}$ of the sub-channel $p$ is $w_{n}^{(p)}=w_{n P+p-1}$. According to multi-channel output vector $\boldsymbol{r}_{n}$, FSE makes a estimation about information source sequence $s_{n}$,

$$
y_{n}=\sum_{i=0}^{L_{\mathrm{w}}} \mathrm{w}_{i}^{T} \mathrm{r}_{n-i}=\boldsymbol{w}^{T} \boldsymbol{r}(n)
$$

Where

$$
\begin{gathered}
\boldsymbol{w}_{n}=\left(w_{n}^{(1)}, w_{n}^{(2)}\right. \\
\left.r_{n-1}, \cdots, \boldsymbol{r}_{n-L_{w}}\right)^{T} .
\end{gathered}
$$

The received signal in the (2) could be expanded to the following form:

$$
\begin{gathered}
\boldsymbol{r}(n)=\left(\boldsymbol{r}_{n}, \boldsymbol{r}_{n-1}, \cdots, \boldsymbol{r}_{n-L_{w}}\right)^{T} \\
\text { The received signal } \\
\boldsymbol{r}(n)=\boldsymbol{H s}(n)+\boldsymbol{v}(n)
\end{gathered}
$$

Where $\boldsymbol{H}$ refers to the channel matrix. The system output could be given when (4) is applied to (3).

$$
y_{n}=\boldsymbol{W}^{T} \boldsymbol{H} \boldsymbol{s}(n)+\boldsymbol{w}^{T} \boldsymbol{v}(n)
$$

Where $\boldsymbol{w}^{T} \boldsymbol{H}$ refers to the system impulse response, which coefficient has a feature that sampling points are at the rate of baud rate. So the multi-channel structure of FSE is equal to a single-channel structure.

\section{FSE Blind Equalization Model}

The multi-channel system model above is the model of FSE in the abstract, many authors have given the structure in practice based on FSE model.

J.K. Tugnait proposed FSE-CMA blind equalization structure in [6], FSE uses coefficient updating algorithm of CMA inverse filter which combines the advantages of fractionally-spaced and the CMA. And then [7] gives general FSE equalization realization structure (Fig. 2), which is based on FSE's multi-channel system model. 


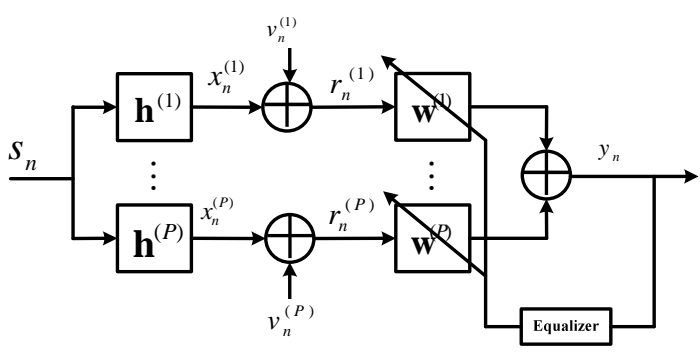

Fig. 2 multi-channel system model of FSE

If the equalization algorithm in Fig. 2 uses CMA, then such equalizer is called FSE-CMA. This is a multi-channel structure and relatively complicated. The single-channel FSE realization structure illustrated in Fig. 3 can be given.

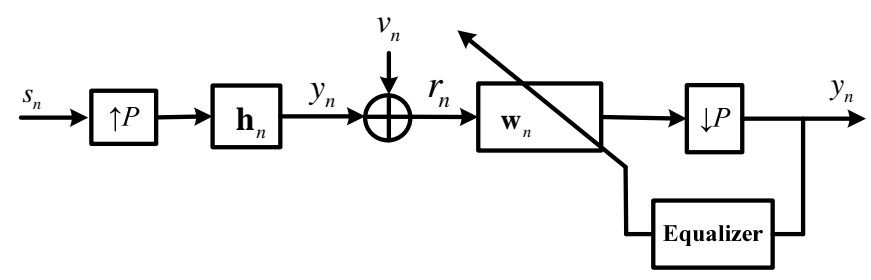

Fig. 3 single channel system model of FSE

Compared with multi-channel model, it is more similar to the structure of SRE, while it also has an extraction module and could be realized easier.FSE-CMA is that FSE adopts the equalization algorithm (coefficient updating algorithm) of CMA, according to the FSE single-channel realization structure $^{[4][8]}$.

\section{Improved Fractionally-spaced Blind Equalization Algorithm}

In order to solve the problem that FSE-CMA cannot track the symbol rate, the improved structure of practical FSE-CMA-Timing, as shown in Fig. 4, is proposed.

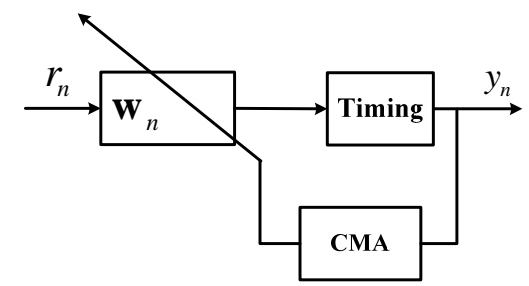

Fig. 4 structure diagram FSE-CMA-Timing

The down-sampling module is replaced with timing module. Timing algorithms have effect on interpolation filter to synchronize signal symbol rate, therefore the inverse filter of FSE only plays a function of equalization. The structure also can be regarded as the combination of equalization and timing algorithms. The inverse filter is located in the forefront of synchronous structure which compensates the channel excellently to make subsequent algorithm could work under no channel distortion circumstance.

Gardner proposed the timing error detection algorithm in the receiver, which is simple and only needs two sampling points for each symbol and one of the two sampling is the best sampling points of symbol (i.e. judge symbols sentence according to this sampling points). Its advantages are nonfacing judgment, and timing recovery is fully independent with carrier phase. Therefore Gardner's Timing synchronization algorithm is adopted in FSE-CMA-Timing.

\section{Performance Simulation and Analysis of FSE-CMA-TIMING}

The convergence speed and performance of FSE-CMA-TIMING is simulated in Matlab. Modulation mode adopts QPSK, 2 sampling points each symbol, the great amplitude is 8192, 
sampling rate is $5 \mathrm{M} / \mathrm{s}$, frequency shift is $25 \mathrm{KHz}$ and phase shift is $0.15 \pi$. The complex channel used in algorithm simulation is expressed as $\boldsymbol{h}=[-0.005-0.004 \mathrm{i}, 0.009+0.03 \mathrm{i},-0.024-0.104 \mathrm{i}$, $0.854+0.520 \mathrm{i},-0.218+0.273 \mathrm{i}, 0.049-0.074 \mathrm{i},-0.016+0.02 \mathrm{i}]$. The structure of the equalizer adopts transverse inverse filter of 15 tap, the initial value of center tap is $1+0 \mathrm{i}$, others are 0 . parameter is selected as $R_{C M A}=134217728$, step length is $\mu=2^{-65}$. Timing algorithms uses Gardner's timing algorithms. The convergence performance of the equalizer is expressed with by remaining intersymbol interference (ISI), the expression of ISI (here use logarithm form) is:

$$
I S I=10 \log \frac{\sum_{k}|h(k) * W(k)|^{2}-|h(k) * W(k)|_{\max }^{2}}{|h(k) * W(k)|_{\max }^{2}}
$$

Symbol * means convolution.

Fig. 5 and 6 illustrate respectively the ISI and constellation of FSE-CMA-Timing when signalto-noise ratio (SNR) is 30dB. Judging from the constellation convergence is very good at high SNR $(30 \mathrm{~dB})$. Since there are frequency shifts, the 7,500 output symbols of FSE-CMA-Timing symbol behave as a circle.

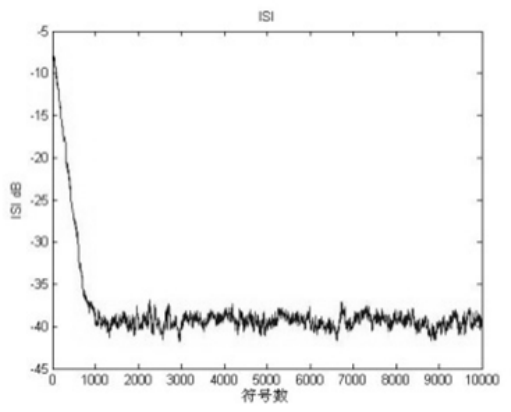

Fig. 5 simulation result of ISI

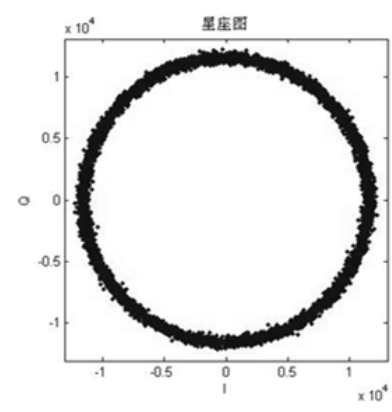

Fig. 6 constellation chart after algorithm convergence

FSE-CMA-Timing convergence is faster, about 500 symbols for QPSK at 30dB can be convergent, and the constellation chart is very clear. Fig. 7 and 8 are given for receiving constellation and FSE-CMA-Timing's output constellation respectively at 10dB SNR.

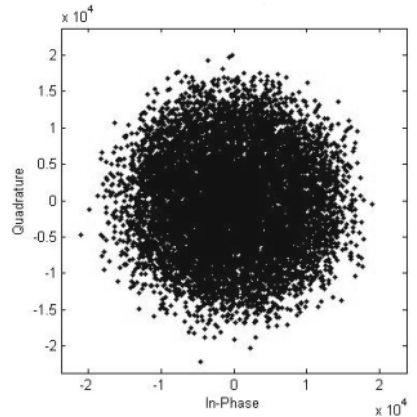

Fig. 7 constellation chart of received signal in SNR 10dB

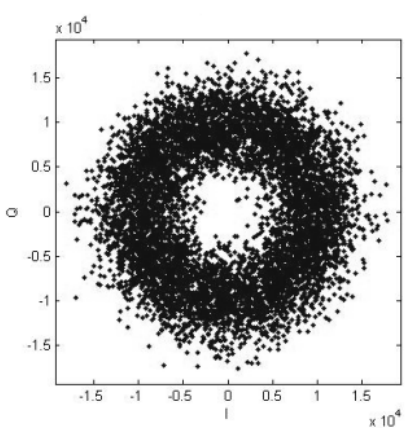

Fig. 8 output of FSE-CMA-Timing in SNR 10dB

Because of the low SNR, white noise makes constellation points thick, while it is still a circle. And compared with receiving signal constellation chart the timing effect still is more obvious.

The inverse filter of FSE-CMA-Timing is fractionally-spaced. In simulation if add channel distortion to the signals, then the ISI of FSE-CMA-Timing wouldn't be calculated. Similarly, if adding channel distortion to fractionally-spaced signal, the ISI of CMA can't be calculated. If adding channel distortion in symbol interval and fractional interval, the actual channel distortion effect is different. The ISI cannot be compared. But the calculation of $e_{s}(k)$ and coefficient of channel and inverse filter is irrelevant, it can be used for the performance comparison between CMA and FSE-CMA -Timing. Steady-state error $e_{s}(k)$ is expressed as 


$$
e_{s}(k)=\left.|| y(k)\right|^{2}-R_{C M A} \mid
$$

When a 7 tap complex channel is supposed and CMA algorithm and FSE-CMA-Timing both use 15 tap inverse filters at the 30dB high SNR and 10dB low SNR respectively, the steady-state error is shown in Fig. 9 and 10. when inverse filter's tap number is 7 at the high signal-to-noise ratio $30 \mathrm{~dB}$ and low SNR 10dB respectively, the steady-state error is shown in Fig. 11 and 12. When inverse filter's tap number is 5 at the high signal-to-noise ratio $30 \mathrm{~dB}$ and low SNR $10 \mathrm{~dB}$ respectively, the steady-state error is shown in Fig. 13 and 14.

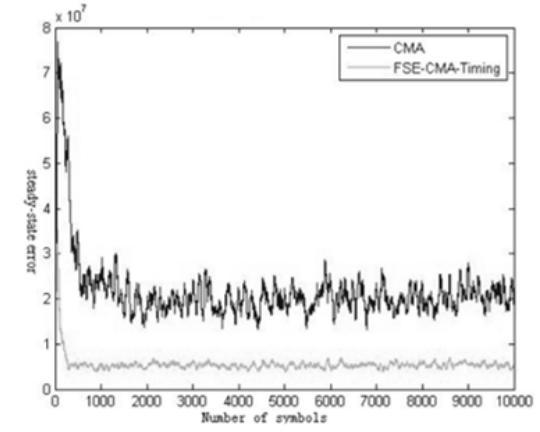

Fig. 9 steady-state error of 15 tap inverse filter in SNR 30dB

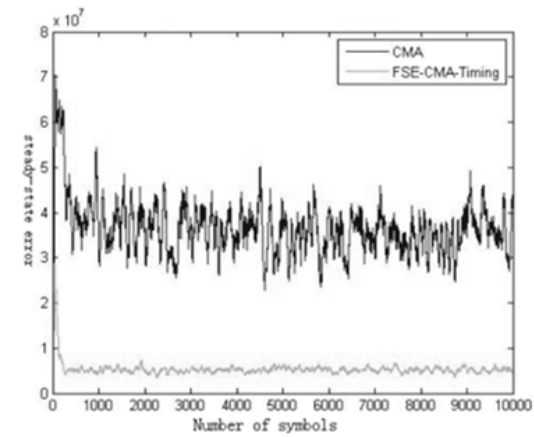

Fig. 11 steady-state error of 7 tap inverse filter in SNR 30dB

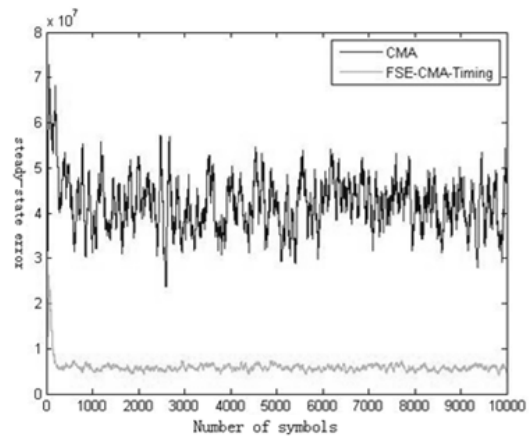

Fig. 13 steady-state error of 5 tap inverse filter in SNR 30dB

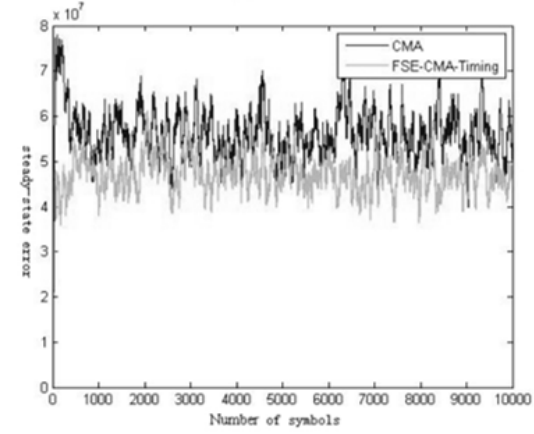

Fig. 10 steady-state error of 15 tap inverse filter in SNR 10dB

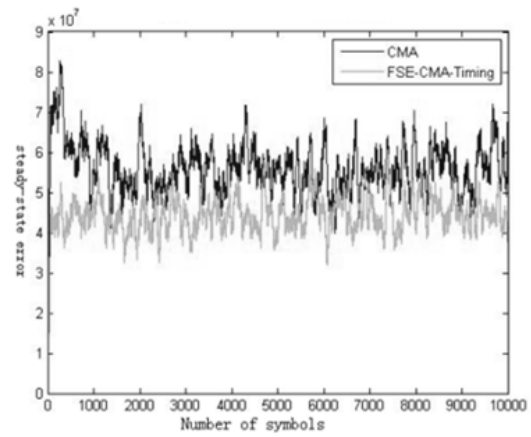

Fig. 12 steady-state error of 7 tap inverse filter in SNR 10dB

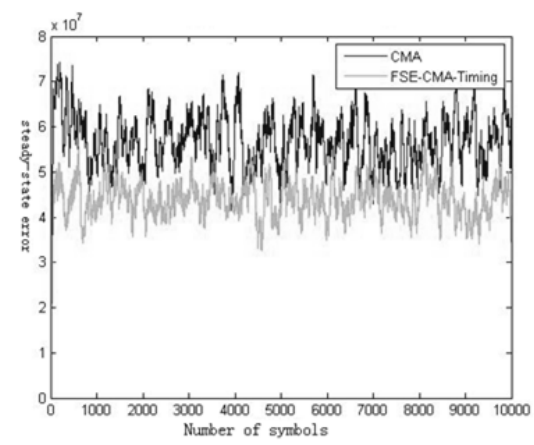

Fig. 14 steady-state error of 5 tap inverse filter in SNR 10dB

The comparison shows that FSE-CMA-Timing is characterized by lower steady-state error. The steady-state error of FSE-CMA-Timing is significantly below to the CMA at high SNR; at low SNR the steady-state error of FSE-CMA-Timing is slightly below the CMA. When equalizer length is less than channel order, FSE-CMA-Timing is significantly better.

\section{Summary}

A joint timing and fractionally-spaced equalization algorithm is proposed in this paper. It adopts Gardner algorithm instead of down-sampling in conventional fractionally-spaced equalization to 
solve the problem of that the filter can't compensate timing error. In the end, the simulation and analysis shows that the proposed algorithm has the higher performance than CMA with different number of inverse filter taps.

\section{References}

[1] Dirk.T.M.Slock, Constantinos. B.Papadias. Blind Fractionally-Spaced Equalization Based on Cyclostationarity[J],IEEE Transon IT,1994.3: 1286-1290.

[2] Sun Shouyu, Zheng Junli, Xu Zhongyong, Zhang Qi. Bind Fractionally Spaced Equalization via Modified Constant Modulus Algorithm[J]. ACTA ELECTRONICA SINICA, 2003,31(11):1732-1735.

[3] Li Song, Ge Lindong. Performance Analysis of Fractionally-Spaced Equalizer Adapted Via the Constant M odulus Algorithm[J]. Journal of Information Engineering University, 2004, 5(2):79-82.

[4] A.Nasir, S.Durrani and R.A.Kennedy, Blind Fractionally Spaced Equalization and Timing Synchronization in wireless Fading Channels[J], in Proc. 2nd International Conference on Future Computer and Communication, vol3, Wuhan, China, pp15-19, 21-24 May,2010

[5] Dirk.T.M.Slock. Blind Fractionally-spaced Equalization, Perfect-reconstruction Filter Banks and Multichannel Linear Prediction[J], IEEE Transon IT,1994.4:585-588.

[6] J.K Tugnait,A Parallel Multimodel CMA/Godard Adaptive Filter Bank Approach To Fractionally-Spaced Blind Adaptive Equalization[J], IEEE Conf on Communications, 1994.4:549553.

[7] J.K.Tugnait. On Fractionally-Spaced Blind Adaptive Equalization under Symbol Timing Offsets Using Godard And Related Equalizers[J]. IEEE Conf on Communications 1995.5: 19761979.

[8] J.R.Trichier, C.R.Johnson. Blind Fractionally-spaced equalization of digital cable TV[C].In Proc.8th IEEE Workshop Statistical Signal Proc. Corfu’ Greece, 1996.122 -130. 